\title{
Classification of Non-ferrous Metals using Magnetic Induction Spectroscopy
}

DOI:

10.1109/TII.2017.2786778

\section{Document Version}

Accepted author manuscript

Link to publication record in Manchester Research Explorer

\section{Citation for published version (APA):}

OToole, M. D., Karimian, N., \& Peyton, A. J. (2017). Classification of Non-ferrous Metals using Magnetic Induction Spectroscopy. IEEE Transactions on Industrial Informatics, 14(8), 3477-3485.

https://doi.org/10.1109/TII.2017.2786778

\section{Published in:}

IEEE Transactions on Industrial Informatics

\section{Citing this paper}

Please note that where the full-text provided on Manchester Research Explorer is the Author Accepted Manuscript or Proof version this may differ from the final Published version. If citing, it is advised that you check and use the publisher's definitive version.

\section{General rights}

Copyright and moral rights for the publications made accessible in the Research Explorer are retained by the authors and/or other copyright owners and it is a condition of accessing publications that users recognise and abide by the legal requirements associated with these rights.

\section{Takedown policy}

If you believe that this document breaches copyright please refer to the University of Manchester's Takedown Procedures [http://man.ac.uk/04Y6Bo] or contact uml.scholarlycommunications@manchester.ac.uk providing relevant details, so we can investigate your claim.

\section{OPEN ACCESS}




\title{
Classification of Non-ferrous Metals Using Magnetic Induction Spectroscopy
}

\author{
Michael D O'Toole, Member, IEEE, Noushin Karimian and Anthony J Peyton
}

\begin{abstract}
Recycling automotive, electronic and other end-oflife waste liberates large quantities of metals which can be returned to the supply chain. Sorting the non-ferrous metals however, is not straightforward. Common methods range from laborious hand-sorting to expensive and environmentally deleterious wet processes. The goal is to move towards dry processes, such as induction sensors and vision systems, which can identify and sort non-ferrous scrap efficiently and economically.

In this paper, we present a new classification method using magnetic induction spectroscopy (MIS) to sort three high-value metals that make up the majority of the non-ferrous fraction copper, aluminium and brass. Two approaches are investigated: The first uses MIS with a set of geometric features returned by a vision system, where metal fragments are matched to known test pieces from a training set. The second approach uses MIS only. A surprisingly effective classifier can be constructed by combining the MIS frequency components in a manner determined by how eddy currents circulate in the metal fragment. An average precision and recall (purity and recovery rate) of around $92 \%$ was shown. This has significant industrial relevance, as the MIS-only classifier is simple, scalable, and straightforward to implement on existing commercial sorting lines.
\end{abstract}

Index Terms-Waste recovery, Recycling, Electromagnetic Induction, Impedance measurement, Classification algorithms, Spectroscopy.

\section{INTRODUCTION}

A sustainable future means moving towards a model where end-of-life products are recycled to become the feedstock of the new. This poses a number of difficult question for industry and legislators; not least, how can the valuable materials be recovered, and the maximum value be extracted from the remainder? A leading example is the automotive industry where every year, over 40 million vehicles worldwide are designated end-of-life and marked for disposal [1]. These vehicles often contain large quantities of steel, aluminium and other valuable metals. Nearly 8 million end-of-life vehicles are generated per year in Europe alone, leading to the European Union issuing directive 2000/53/EC, imposing a target of $85 \%$ recycle and reuse rate for end-of-life vehicles by 2015 [2]. The most recently available statistics show that seventeen member states had achieved this goal as of 2014 [3].

The disposal of end-of-life vehicles involves the removal of pollutants (oils, batteries, etc.), then shredding in to smaller fractions from which the metal content is extracted [4], [5].

Manuscript received August 2, 2017; revised October 17, 2017; accepted December 4, 2017.

The authors are with the School of Electrical and Electronic Engineering, The University of Manchester, M13 9PL, Uk (e-mail: michael.otoole@manchester.ac.uk, noushin.karimian@manchester.ac.uk a.peyton@manchester.ac.uk)
Ferrous metals are the most easily recovered and are profitably recycled [5]. The non-ferrous fraction is mainly composed of aluminium alloys (approx. 78\%), followed by smaller proportions of brass (12\%), and copper (5\%) [6], [7]. This fraction is much more difficult to sort and recover.

One technique is dense-media separation, where fine particles of magnetite or ferrosilicon are suspended in water to create a slurry of controlled specific gravity. Low density metals, e.g. aluminium, float to the surface whereas heavier ones sink [8]. Maintaining these slurries, and water treatment, is expensive and has a significant environmental impact [8], [9]. The method is also ineffective at sorting metals where the difference in density is small, such as copper from brass.

Dry methods are an alternative. The most basic is to sort the metals by hand using colour [9]. This works well where the colour is distinctive, e.g. white from red metals, but the process is dependent on low labour costs. Kutil et al. [10] demonstrated an autonomous colour sorting system, however accuracy was diminished by machine-vibration, variations in ambient lighting, reflections and other artefacts generated under industrial conditions. Multi-spectral vision systems have shown significant promise. These are capable of separating materials with similar colour properties, and have reported recovery rates of 95\% [11] and 98\% [12] in the literature. However, they have yet to be tested under industrial conditions, e.g. with dirty samples and fast belt speeds, which will most likely degrade performance. Other methods include laser induced breakdown spectroscopy (LIBS) and X-ray beam absorption [13]. These are the gold-standard in metal identification but are expensive, and can be difficult to adapt practically for high-throughput and harsh industrial environments.

We propose a method using magnetic induction spectroscopy (MIS) for classifying non-ferrous metals. MIS is the change in the magnetic field induced in an object, in response to different frequency excitation magnetic fields. To explain, consider a conductive sphere in free space, centred at the origin, with radius $a$, conductivity $\sigma$ and permeability $\mu=\mu_{0}=4 \pi \times 10^{-7}$ (free space). The sphere is illuminated by a uniform oscillating magnetic field acting along an axis $Z$. This induces eddy-currents to circulate in the object, which in turn induces a secondary or scattered magnetic field.

Let us take a point $z$ along the Z-axis some distance outside of the sphere $(z>a)$. Denote $H_{e x}$ and $H_{s}$ as the complex component in the $\mathrm{Z}$ direction of the excitation and secondary magnetic field respectively, at the point $z$ and at frequency $f$. The scattered field is given by the well-known analytical 
expression [14], [15],

$$
\frac{H_{s}}{H_{e x}}=-\frac{3 a^{3}}{z^{3}}\left(\frac{1}{\alpha^{2}}+\frac{1}{3}-\frac{\cosh \alpha}{\alpha \sinh \alpha}\right)
$$

where $\alpha=(i 2 \pi f \sigma \mu)^{1 / 2} a$.

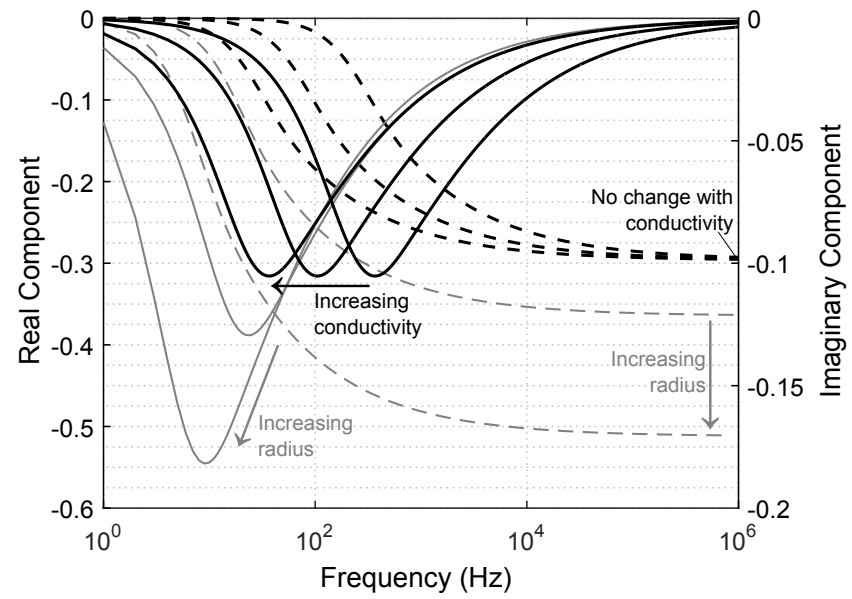

Fig. 1: The magnetic induction spectra of a sphere $\left(H_{s} / H_{e x}\right)$, with real (- -) and imaginary components (-). Black lines show the effect of changing conductivity $\sigma$ and grey lines the effect of changing radius $a$

Figure 1 shows the real and imaginary component of $H_{s} / H_{e x}$ over a frequency range $f \in\left(1,10^{6}\right) \mathrm{Hz}$, for a sphere with radius $a \in\{20,25,40\} \mathrm{mm}$ and conductivity $\sigma \in\{10,35,100\} \mathrm{MS} / \mathrm{m}$. This plot is the magnetic induction spectra of the sphere. The real component is shown to be a sigmoid shape converging to an asymptote as $f \rightarrow \infty$. The imaginary component shows a curve which decreases to a minima then tend towards zero. This characteristic shape is typical of non-magnetic metal objects - not just spheres - see for example [16], [17], [18].

Intuitively, the shape of the spectra can be explained in terms of the skin-depth effect, i.e. the tendency for eddycurrents to distribute closer towards the surface as frequency increases. This reduces the cross-section through which current can flow, and consequently increases resistance in the object. This effect becomes significant when the skin-depth reaches some fraction of the characteristic dimension of the object, resulting in the minima seen on the imaginary component. Increasing the conductivity shifts the curves left as skin-depth becomes significant at lower frequencies. The asymptote of the real component does not change with conductivity. At high frequencies, the eddy-currents only flow along the surface of the object and thus are unaffected by the conductivity. Changing the radius on the other hand does affect the asymptote. The surface geometry is altered changing the path which the eddy-currents flow. Increasing the radius moves the asymptote downwards in this case.

In this paper, we introduce a new method to classify the three metals predominant in the non-ferrous fraction; copper, aluminium and brass. We use: (1) the magnetic induction spectra of the metal fragments combined with geometric features returned by a vision system, and (2) the magnetic induction spectra alone based on an understanding of how the eddy-currents circulate at different frequencies. This work is the first to demonstrate MIS as an information-rich feature source for use in metal recovery, and first to propose a classification method using this new feature set based on an understanding of the physics of magnetic fields. As a method, it is simple, practical, fast and scalable. It is straightforward to implement on a commercial sorting line, and capable of the high-throughput operation demanded by industry.

\section{METHOD}

\section{A. ShredderSort MIS + VIA System}

The MIS + VIA (Vision Image Analysis) System was developed by a consortium for the EU project ShredderSort (No. 603676) to demonstrate new sorting algorithms on an industrial-scale and under realistic operating conditions. The system is shown in figure 2. It consists of a $1 \mathrm{~m}$ wide conveyor with air ejectors at the discharge-end (Regulator Cetrisa, Spain and Lenz Instruments, Spain), a high speed vision image analysis system (VIA) (Joanneum Research, Austria), and a magnetic induction spectroscopy sensor [19] (University of Manchester, UK). Signal processing and operation of the system is conducted from the Interface and Control cabinet (Lenz Instruments, Spain).

A metal sample deposited at the in-feed end of the conveyor will first pass the VIA system. This uses structured-laser light to determine size, shape, position and orientation of the test pieces and return a set of geometric features. The system can return up to 24 different features, however in the present work, we will only use the following most useful:

- $f_{1}$ : Area of the test piece $\left(\mathrm{mm}^{2}\right)$.

- $f_{2}$ : Minimum feret diameter $(\mathrm{mm})$.

- $f_{3}$ : Maximum feret diameter $(\mathrm{mm})$.

- $f_{4}$ : Mean height (mm).

The metal sample then passes over the sensitive zone of the MIS sensor. This returns a six point spectra at frequencies around 2, 4, 8, 16, 32 and $64 \mathrm{kHz}$. Signal processing for the MIS system is on a NI PXI-1033 chassis with 2x PXI7853R I/O module (National Instruments, USA) housed in the interface and control cabinet. This system contains a number of ADCs and DACs to output the excitation waveforms and measure the receive signals, and an FPGA for demodulation. To aid ongoing discussion in this paper, we will designate the demodulated real and imaginary components of the spectra as,

$$
Z_{p}^{\prime}+i Z_{p}^{\prime \prime}
$$

where the subscript $p$ denotes the frequency point, i.e. $p \in$ $P:=\{2,4,8,16,32,64\} \mathrm{kHz}$. Note that this is a relative measurement as it is not scaled to give true $H_{s} / H_{e x}$. The NI PXI-1033 also reads the belt-encoder on the conveyor system and controls the air ejectors. The latter were not used in the present work. Classification and overall control of the MIS + VIA system is performed on a desktop PC located in the interface and control cabinet. 


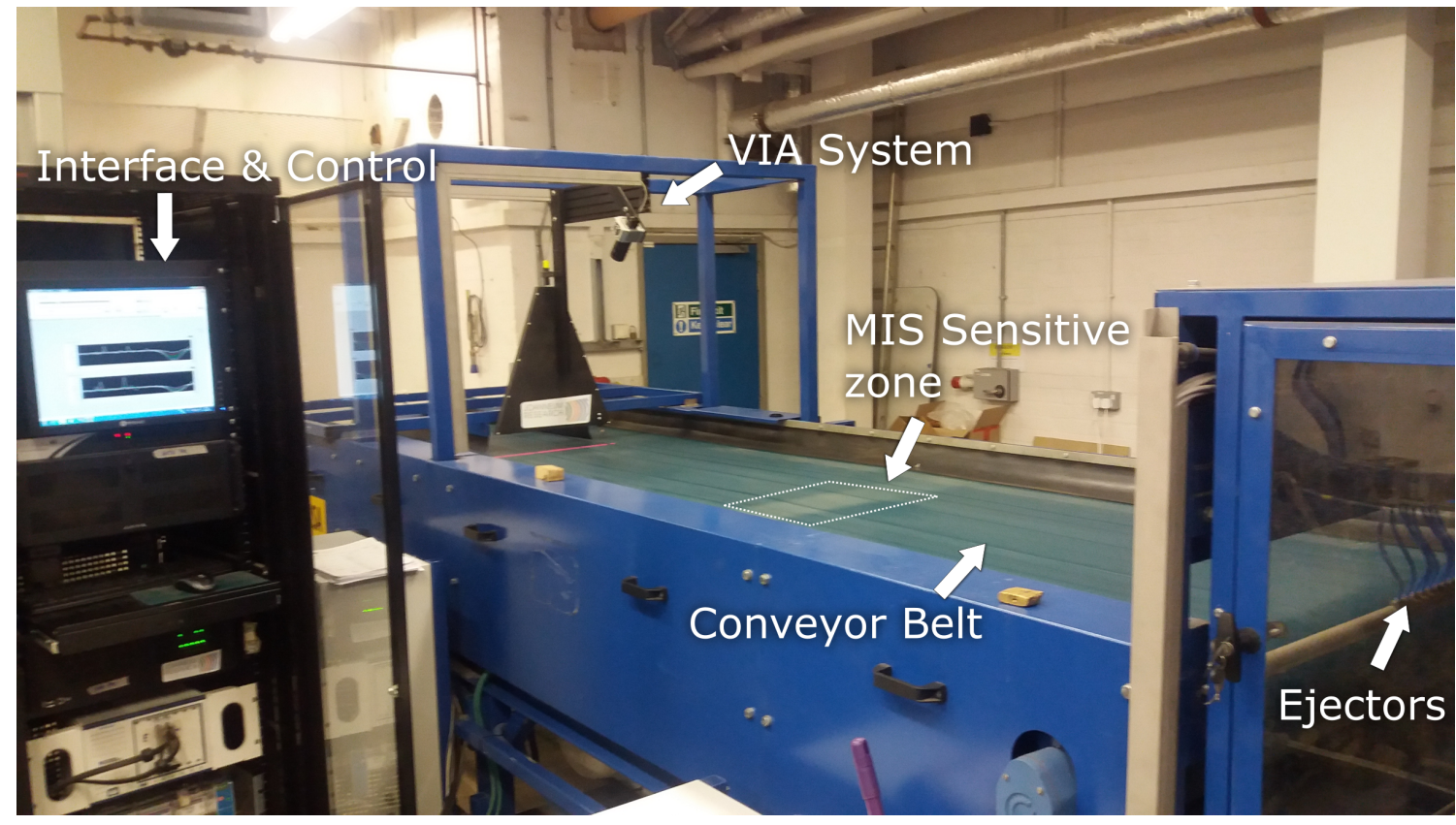

Fig. 2: SHREDDERSORT MIS + VIA System

\section{B. Magnetic Induction Spectroscopy (MIS) Sensor}

The MIS sensor is shown in figure 3 . It consists of an aluminium enclosure $(750 \mathrm{~mm} \times 1220 \mathrm{~mm} \times 207 \mathrm{~mm})$ with a stepped acetal lid, mounted underneath a conveyor belt, leaving a gap of approximately $5 \mathrm{~mm}$ between belt and sensor. The enclosure contains an array of sixteen solenoids, and power and instrumentation amplifiers situated in compartments fore and aft of the array. Each solenoid is used to transmit a multi-frequency excitation and detect the resultant secondary magnetic field as the metal targets pass.

The cross-section of a solenoid is shown in figure 4. It consists of, (1) an internal core with a coil to generate the excitation magnetic field, and (2) an outer sleeve with two coils to detect the secondary field. The internal core is constructed from an acetal tube with two $6 \mathrm{~mm}$ diameter ferrite rods (Fair-Rite 4077276011) pressed inside. A 64-turn coil is wrapped in two sections around the tube over the ferrite cores using $0.4 \mathrm{~mm}$ enamelled wire. This coil is driven by a power amplifier (LT1210, Linear technologies). The outer sleeve is also constructed from an acetal tube with insets cut to a $16 \mathrm{~mm}$ diameter. A 600 -turn coil of $0.2 \mathrm{~mm}$ enamelled wire is wrapped around each inset. The coils are wound in opposing directions and joined to form an axial gradiometer. This can be finely balanced using a lead-screw at the bottom of the solenoid. The gradiometer is then connected to an instrumentation amplifier (AD8029, Analog Devices).

The solenoids are spread evenly over a $400 \mathrm{~mm}$ width across the centre of the conveyor in three rows approximately $90 \mathrm{~mm}$ apart in a diagonal pattern. This creates sixteen $25 \mathrm{~mm}$ wide channels, with a solenoid covering each channel. To minimise potential cross-talk between solenoids, each one transmits an excitation at a different frequency-set to its neighbours. The frequency set $P$ is shifted in the spectrum slightly to create four different multi-sine waveforms. These are then assigned

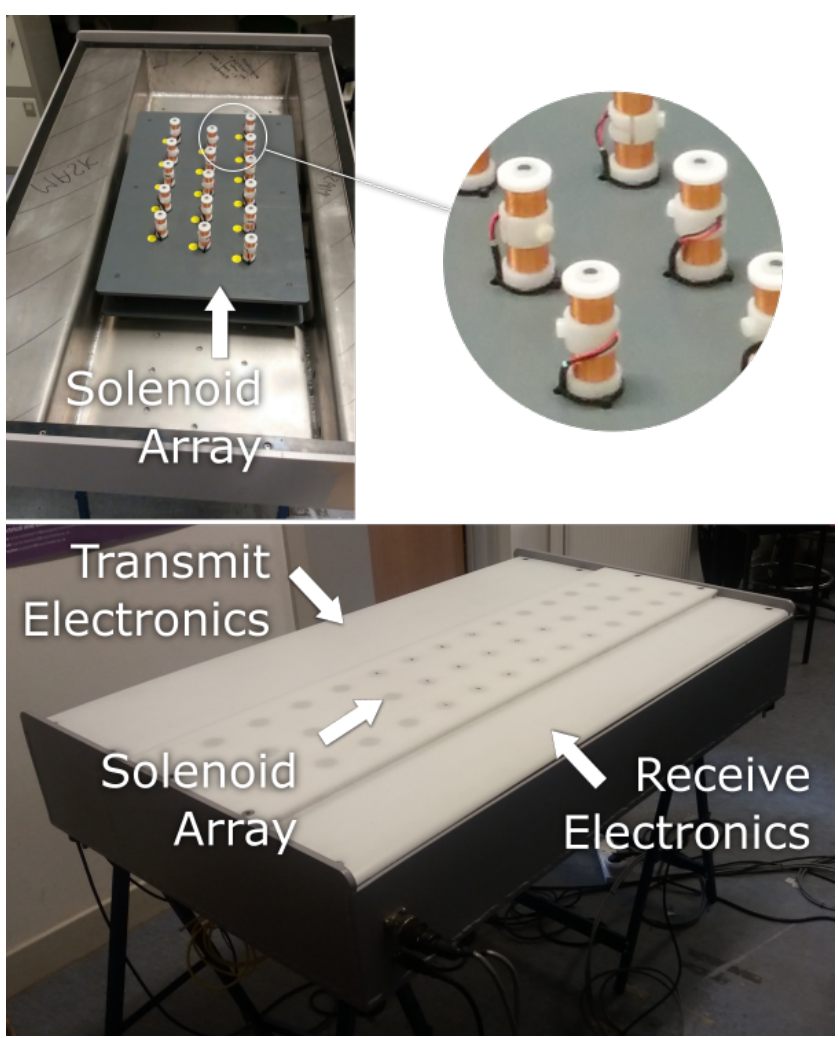

Fig. 3: MIS Sensor

to each solenoid in such a way that no two adjacent solenoids have the same waveform.

Each solenoid is calibrated using a ferrite cylinder (Ferroxcube, 4B1) with diameter $10 \mathrm{~mm}$ and height $20 \mathrm{~mm}$, in the manner described here [20]. The ferrite is placed on the conveyor-belt coincident with the central axis of a solenoid. 


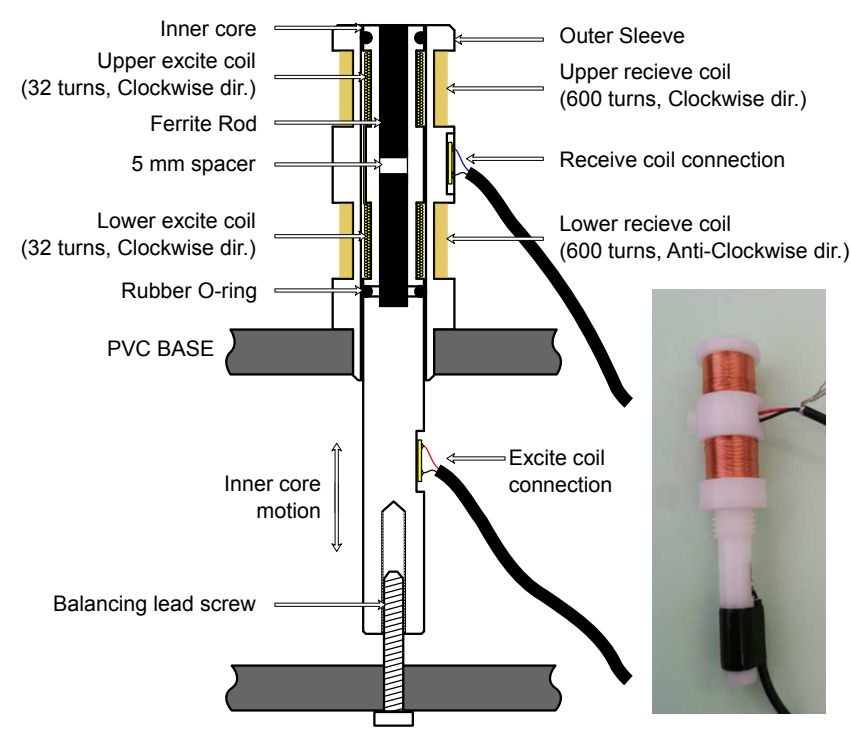

Fig. 4: Solenoid Schematic

Measurements are calibrated to give $Z_{p}^{\prime}+i Z_{p}^{\prime \prime}=1$ over all frequencies. This calibration procedure accounts for any phase-shifts or delays present in the system.

In what follows, we only collect data from a single solenoid of the array. A guide is deployed at the infeed end of the conveyor to guide the test pieces over the specific solenoid. While the solenoids are essentially duplicates, there may be slight differences in their responses due to calibration or manufacturing errors. Confining our measurements to a single solenoid ensures the collection of the most robust and reliable dataset without loss of generality in the approach.

\section{Test Pieces and Data Collection}

A total of 117 test pieces were used with 36 copper pieces, 44 aluminium, and 37 brass. An example set representative of the test pieces is shown in figure 5. The pieces were cut to different sizes and shapes from stock metals, such as bar, rod, channel, etc., provided by a local metal supplier (Manchester Metals, UK). The metals were common grades or alloy types typically used in manufacture. The size specification for the samples is height from $3 \mathrm{~mm}$ to $25 \mathrm{~mm}$, and width and length from $15 \mathrm{~mm}$ to $50 \mathrm{~mm}$.

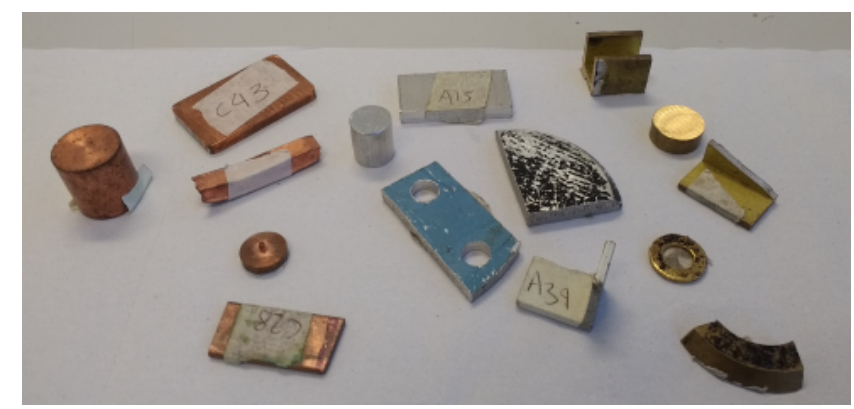

Fig. 5: Sub-set of typical test pieces. A total of 117 test pieces are used in copper (36 pieces), aluminium (44) and brass (37).

Data on each test piece was collected using the following procedure: The system is activated and allowed to run for several minutes to obtain a stable operating temperature. The conveyor belt is set to run at $1 \mathrm{~m} / \mathrm{s}$. Each piece is assigned a numerical designation and is dropped on the conveyor belt in order. The piece is channelled to run over a specific solenoid using the guide. It passes across the structured laser of the vision system and its geometric features are obtained and recorded. The piece then passes over the magnetic induction sensor, where the real and imaginary components at the six frequencies are recorded at a $250 \mathrm{~Hz}$ sample-rate. This gives a spatial resolution (travel of test piece between samples) of $4 \mathrm{~mm}$ on a $1 \mathrm{~m} / \mathrm{s}$ conveyor. A single complex value for each frequency is obtained by taking the point where the magnitude of the response is maximum. Any misses due to the vision system not detecting the object, or because it moved outside of the channel, were marked and the measurement repeated.

\section{Classification}

We propose classification based on two different feature sets: The first uses the magnetic induction spectra combined with geometric properties of the test piece returned by the vision image analysis system. The second is simpler. It uses only MIS, but where the features are chosen based on an understanding of how eddy-currents circulate in the test piece.

1) Algorithm 1, MIS + geometric properties: A simple sample matching classifier similar to a nearest neighbour approach is proposed. The aim is to first match the test-piece to pieces in the training set with similar geometric features, producing a 'nearest-match' for each class. Then, the MIS of the test piece is compared to the nearest-matching pieces for each class and assigned the class that has the most similar spectra. Formally:

1) Recall that $Z_{p}^{\prime}$ and $Z_{p}^{\prime \prime}$ are the real and imaginary components at frequency $p \in P$ of a test piece, and $\left\{f_{1}, f_{2}, \ldots\right\}$ its set of geometric features (see section II-A). Denote $\hat{Z}_{p}^{\prime}$ and $\hat{Z}_{p}^{\prime \prime}$ and $\left\{\hat{f}_{1}, \hat{f}_{2}, \ldots\right\}$ as the same for a piece drawn from the training set.

2) Normalise the geometric features (test and training) such that they have zero mean and unity variance [21]. We use the prime ' to indicate the feature has been normalised,

$$
f_{j}^{\prime}=\left(f_{j}-\mu_{j}\right) / s_{j}, \quad \hat{f}_{j}^{\prime}=\left(\hat{f}_{j}-\mu_{j}\right) / s_{j}
$$

where $\mu_{j}$ and $s_{j}$ is the mean and standard deviation for feature $j$ of all pieces in the training set.

3) Find amongst the training set of pieces belonging to class $i$ the first $K$ pieces with the nearest matching geometric features to the test piece. The nearest matching training piece is the one with the smallest $d$,

$$
d=\sqrt{\sum_{j=1}^{4}\left(f_{j}^{\prime}-\hat{f}^{\prime}{ }_{j}\right)^{2}} .
$$

We refer to the set of $K$ nearest-matching training pieces for class $i$ as $U_{K}^{i}$.

4) Calculate the mean-squared difference between the real and imaginary components of the spectra for each piece in $U_{K}^{i}$ and the test piece, then take the minimum. In 
this manner we create a function $E_{i}$ for each class $i$ as follows,

$$
E_{i}=\min _{j \in U_{K}^{i}}\left(\sum_{p \in P} \frac{\left(Z_{p}^{\prime}-\hat{Z}_{p, j}^{\prime}\right)^{2}+\left(Z_{p}^{\prime \prime}-\hat{Z}_{p, j}^{\prime \prime}\right)^{2}}{|P|}\right)
$$

where $|P|$ is the number of measured frequencies.

5) The test piece is assigned a class by evaluating $E_{i}$ for each class and,

$$
\text { Class := } \arg \min \left\{E_{C u}, E_{A l}, E_{B r}\right\}
$$

2) Algorithm 2, MIS only: To establish the rationale for a purely MIS approach, we make two observations about the characteristic shape of the magnetic induction spectra for a given non-magnetic conductive test piece following the discussion in section I:

1) The asymptote of the real component $Z_{p}^{\prime}$ as $p \rightarrow \infty$ is equivalent to treating the test piece as a perfect electrical conductor, i.e. eddy-currents only circulate on the boundary. Therefore, $Z_{p}^{\prime}$ becomes independent of conductivity as it approaches the asymptote but retains its dependence on shape and size.

2) Any point on the curve of the imaginary component $Z_{p}^{\prime \prime}$, that is not too close to the zero asymptote, is a function of conductivity, and size and shape of the test piece.

We suggest that by comparing the two MIS components of the test piece as described, with one sensitive and the other insensitive to conductivity, we may account for the effects of size and shape of the test piece and classify its material using only magnetic induction measurements. This idea was initially proposed in previous work by the authors [22]. This work presented some preliminary results for a small set of manufactured samples (36 pieces in total) on a laboratorybased test rig. The results were generally positive, but not conclusive given the small sample set. In this work, we expand on this idea and provide more conclusive evidence with a large sample-set and the industrial-scale demonstrator described.

This is a novel approach. Multi-frequency excitation and measurement creates a new, information-rich set of features for classification despite modest changes to industrial infrastructure. Induction sensors are already in use commercially, e.g. Steinert KSS and ISS systems (Steinert GmbH, Germany). Our work extends their use by an inventive analysis of the processed signals. We also identify how different parts of the spectra express characteristics about the object. In what follows, we will compare the two components described and determine an algorithm for separating the three metals.

\section{RESULTS AND DISCUSSION}

\section{A. Design of MIS Classifier}

A typical magnetic induction spectra for one of the test objects is shown in figure 6. The figure shows part of the characteristic profile described in section I: The imaginary component $Z_{p}^{\prime \prime}$ is converging towards zero and the real component $Z_{p}^{\prime}$ converging to an asymptote at around -0.33 . $Z_{64 k \mathrm{~Hz}}^{\prime}$ is close to the asymptote and it is therefore realistic to

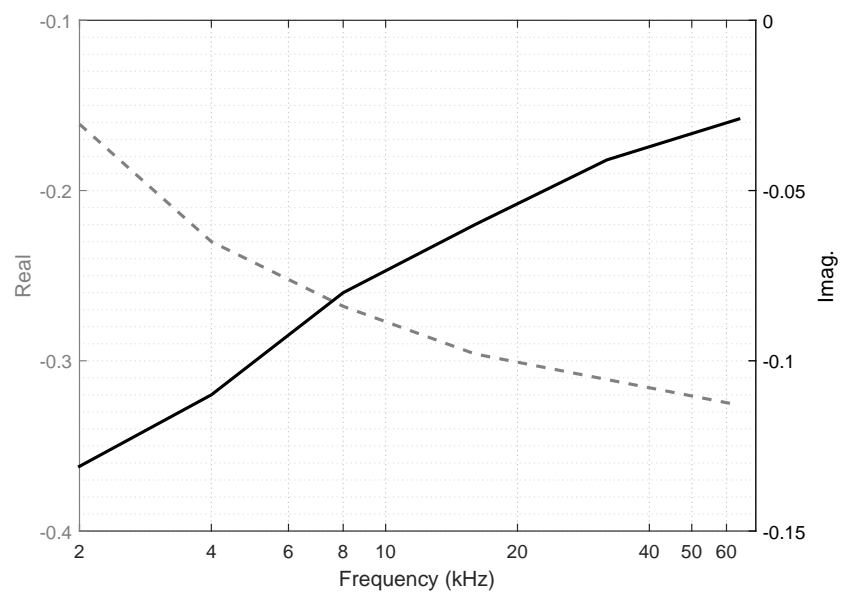

Fig. 6: Spectral response of a rough aluminium cuboid, dimensions $47 \mathrm{~mm} \times 24 \mathrm{~mm} \times 6.5 \mathrm{~mm}$, with $(--)$ the real component $Z^{\prime}$, and (-) the imaginary component $Z^{\prime \prime}$.

assume that it will be insensitive to conductivity, satisfying the assumption discussed in section II-D.

Figure 7 compares the imaginary component at 2, 4, 8 and $16 \mathrm{kHz}$ to the real component at $64 \mathrm{kHz}$, or $Z_{64 k \mathrm{~Hz}}^{\prime}$ (close to the asymptote). Each plot shows evidence of the samples banding into three metal classes in order of conductivity, with the highest conductivity (copper) banding towards the top and the lowest (brass) towards the bottom. A measurement at 32 $\mathrm{kHz}$ is also taken however, the imaginary component is close to zero at this frequency and thus provides little insight.

The banding holds well for test pieces with smaller $Z_{64 k \mathrm{~Hz}}^{\prime}$ but appears to break-down, or begin to break-down, after $Z_{64 k \mathrm{~Hz}}^{\prime}<-1.2$. This is acute in figure $7 \mathrm{~d}$ where both copper and aluminium samples depart from the overall trend. This is due to poor quality of measurements, most likely clipping or saturation in the receive electronics caused by too high gain. The coherency of the bands may therefore be improved with greater dynamic range.

The dashed lines in figure 7 are logistic-functions of the form,

$$
Z_{p}^{\prime \prime}=\frac{k_{1}}{1+\exp \left(-k_{2} Z_{64 k H z}^{\prime}\right)}+\frac{k_{1}}{2}
$$

where $p$ depends on the figure, and $k_{1}, k_{2}$ are constants that define the shape of the curve. The logistic-function provides a good description of the shape for each band using only two shape-defining parameters. We propose to use equation (4) to construct a simple classifier to determine the metal type of the test pieces using only spectral information.

In what follows, we examine two logistic-function classifiers using $Z_{4 k \mathrm{~Hz}}^{\prime \prime}, Z_{64 k \mathrm{~Hz}}^{\prime}$ and $Z_{8 k \mathrm{~Hz}}^{\prime \prime}, Z_{64 k \mathrm{~Hz}}^{\prime}$ as features. These two appear to show the clearest banding overall in figure 7.

Define a function for each class $i \in\{C u, A l, B r\}$,

$$
C_{i}=\frac{k_{i, 1}}{1+\exp \left(-k_{i, 2} Z_{64 k H z}^{\prime}\right)}+\frac{k_{i, 1}}{2}-Z_{8 k H z}^{\prime \prime}
$$

The term $C_{i}$ may be considered a distance measure. Any object which falls on the line defined by the logistic-function results 


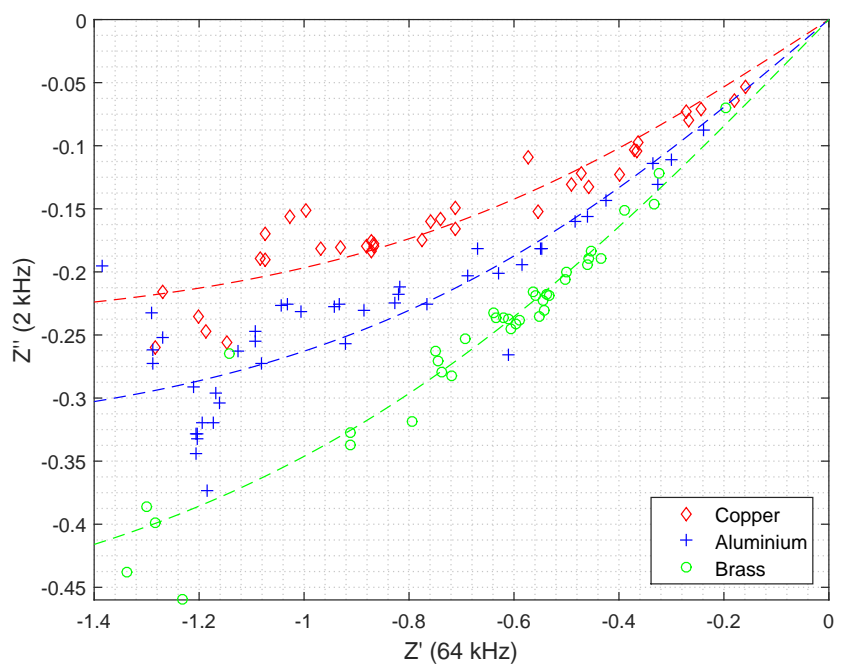

(a)

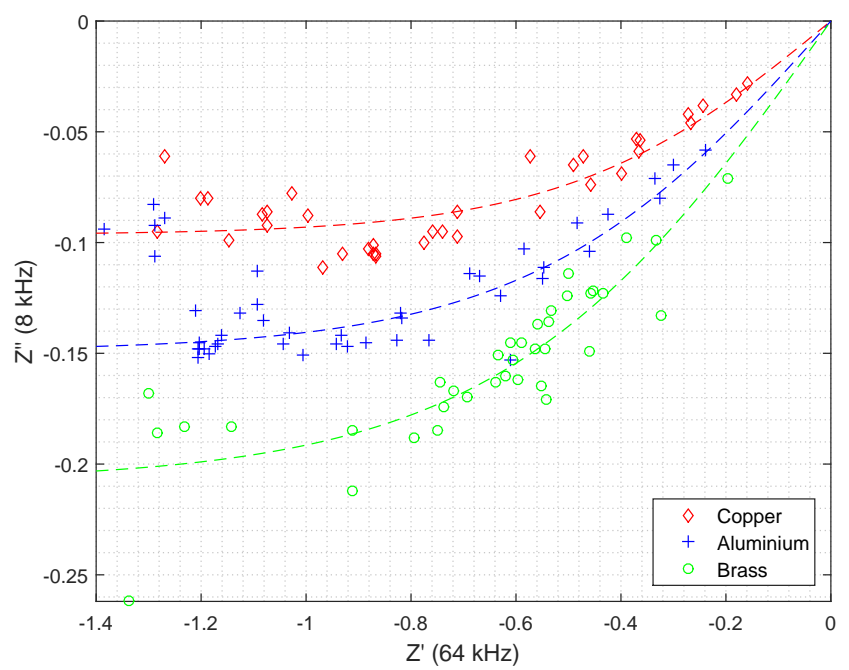

(c)

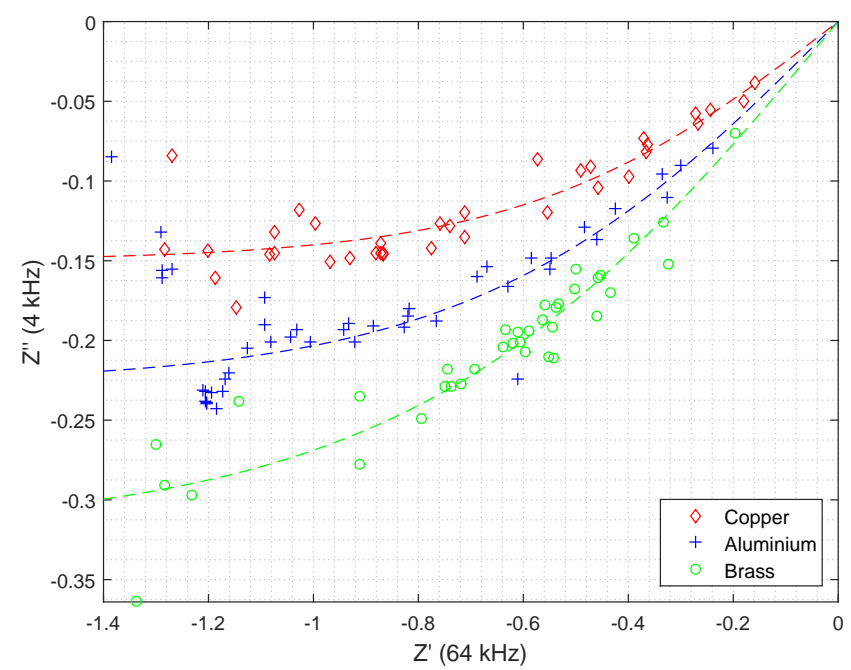

(b)

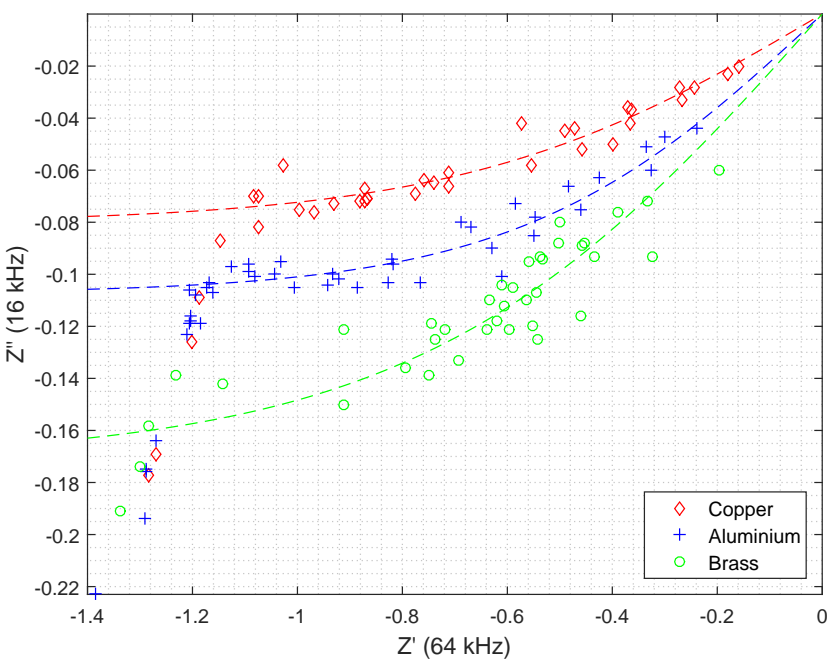

(d)

Fig. 7: Comparison of the imaginary component of the spectra $Z^{\prime \prime}$ at frequencies $2,4,8$ and $16 \mathrm{kHz}$ with the real component $Z^{\prime}$ at $64 \mathrm{kHz}$.

in $C_{i}=0$. A test piece is then assigned to the class with the smallest $C_{i}$. Formally,

$$
\text { Class := } \arg \min \left\{C_{C u}, C_{A l}, C_{B r}\right\}
$$

A classifier for $Z_{4 k H z}^{\prime \prime}, Z_{64 k H z}^{\prime}$ is simply obtained following the same procedure with $Z_{8 k H z}^{\prime \prime}$ replaced with $Z_{4 k H z}^{\prime \prime}$.

The choice of frequency for the imaginary component $Z^{\prime \prime}$ is a difficult problem which merits some discussion. Returning to the spectra of a sphere in figure 1, we see evidence of two different electromagnetic effects. The first effect shows $Z^{\prime \prime}$ decreasing towards a minima as frequency increases. This is due to the relation between frequency and field strength predicted by Maxwell's equations for a linear media. The second effect shows $Z^{\prime \prime}$ increasing and tending towards zero. This is due to the skin-depth effect, where the eddy-currents progressively penetrate less of the object as frequency increases. The minima in the spectra is a point of transition, where one effect becomes dominant over the other.
We desire to take measurements where the same effect is dominant across all the test pieces. Otherwise, the different effects may follow different trends when comparing with $Z_{64 k \mathrm{~Hz}}^{\prime}$. In the present work, measurements are mostly taken from where the skin-depth is dominant - as indicated by figure 6 . We also desire measurements with a good signal-to-noise ratio. If the frequency for $Z^{\prime \prime}$ is too high, the measurement will be small and suffer adversely from noise. A suitable frequency for $Z^{\prime \prime}$ may therefore be chosen by the following heuristics:

1) A frequency sufficiently high such that the skin-depth effect is dominant for $Z^{\prime \prime}$ of the least conductive test piece with the smallest single dimension.

2) A frequency sufficiently low such that $Z^{\prime \prime}$ of the most conductive test pieces can be measured with a good signal-to-noise ratio.

How realistic is it to apply this heuristic in scrap metal sorting? Shredded waste is routinely sorted into size, and the conductivity of the target metals is known. This means 
a reasonable estimate can be made for the frequency of $Z^{\prime \prime}$ which will hold over the fraction to be sorted provided the size criteria of the fraction is sufficiently specific.

\section{B. Classification Performance}

The Leave One Out (LOO) method is used to generate performance statistics on the three classification algorithms described - the two logistic-function based methods, using different frequency components as input features, and the sample matching classifier outlined in section II-D. The LOO method works by removing a single test piece from the data set, then use the remaining pieces to train the classifier. The excluded test piece is then assigned a class, using the newly trained classifiers, and returned to the data set. A second test piece is then removed and the process repeated until each piece has been classified.

Analysis and data processing is performed in Matlab (Mathworks Inc, USA), with training of the logistic-functions (parameters $k_{i, 1}, k_{i, 2}$ ) using Matlab's fminsearch optimisation function. Some outliers are excluded when training the logistic-functions as they significantly skew the best-fit approximation. However, they are retained as test pieces and their classification, or mis-classification in this case, is included in the overall statistics. No outliers are excluded from the matching classifier's training sets. The outliers are chosen as any aluminium piece with $Z_{64 k \mathrm{~Hz}}^{\prime}<-1.2$ and any brass piece with $\left(Z_{4 k H z}^{\prime \prime}<-0.35\right) \vee\left(Z_{8 k H z}^{\prime \prime}<-0.25\right)$. This excludes a total of six pieces from the training sets $(5 \mathrm{Al} ., 1 \mathrm{Br}$.).

Classification results are shown in table I. Table headings are defined according to Soklova et al. [23] and are included in the appendix. Overall the results appear strong, with average accuracies for the MIS-only methods close to $95 \%$, with around $92 \%$ precision and recall. The matching classifier shows relatively poor performance with average accuracy, precision and recall of $83.5 \%, 75.2 \%$ and $75.2 \%$ respectively. This is a surprising result. The classifier works using a two stage matching process: First, a number of training pieces with similar shapes and sizes to the test piece are selected from each class. The test piece is then assigned the class of the selected piece with the most similar MIS. It was expected that this approach would outperform the MIS-only method, as it compares similar pieces, which should have similar MIS.

\section{Geometric Paramaters vs. $Z_{64 k \mathrm{~Hz}}^{\prime}$}

A possible cause of the inferior results for the matching classifier is that the representation of the geometry of the test pieces as features $\left(f_{1}, f_{2}, f_{3}, f_{4}\right)$ is overly reductive. The matching classifier is essentially making a prediction about the magnetic induction spectra for a given set of geometric features and an assumed conductivity. For a test piece with certain geometric features, we are assuming the spectra has one form if it is copper, and another if it is brass. However, if too much information is lost in condensing the geometry to a small set of scalar values, then correlation between shape and magnetic response will be poor, and we can no longer make meaningful predictions. Other possible causes are the quantity of training data being too sparse to populate the feature space,

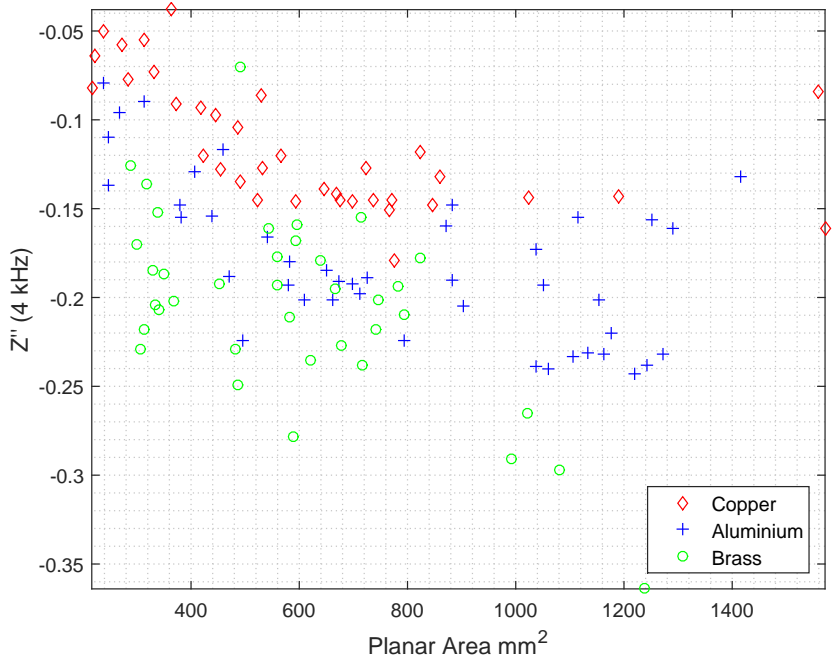

Fig. 8: Comparison of the imaginary component of the spectra at $4 \mathrm{kHz}\left(Z_{4 k H z}^{\prime \prime}\right)$ compared to $f_{1}$, the area of the test piece.

or small variations in the path as pieces pass over the solenoid. The same piece travelling close to the solenoid axis would have a slightly different magnetic response than if it were to traverse off-centre. This might be enough to degrade the performance of the classifier, especially for smaller pieces.

It is notable that using $Z_{64 k \mathrm{~Hz}}^{\prime}$ as a proxy for geometry in the MIS only classifiers outperforms actual geometric features, as used in the matching classifier. For instance, let us compare $Z_{4 k H z}^{\prime \prime}$ (a function of both geometry and conductivity) against a geometric feature. This is shown in figure 8 , using $f_{1}$ or area of the test pieces as returned by the vision system. There is weak banding of the different metal types, with copper predominately at the top, aluminium in the middle, and brass mostly at the bottom of the figure. It is considerably more scattered than figure 7 . The other features $f_{2}, f_{3}, f_{4}$ showed similar or worse results. Their plots are omitted for the sake of brevity. We posit that $Z_{64 k \mathrm{~Hz}}^{\prime}$ better accounts for the variable geometry of the test pieces, or variations in orientation or travel path as the piece passes over the solenoid, as it is itself a product of the eddy-current and magnetic field interactions taking place in the test piece.

\section{Comparison with other Classifiers}

The performance of the classifiers compares favourably to the published literature. Recovery-rates (equivalent of recall) are typically in the range of $90-97 \%$ for X-ray techniques, 80 97\% for LIBS and 86-95\% for optical methods and [13]. X-ray and LIBS sorting is noted for being expensive, in contrast to induction sensors, and are difficult to justify economically.

Kutila et al. [10] reported average precision of $83.5 \%$ using a chromatic plus induction method to sort white (aluminium) from red metals (copper and brass). The precision for separating the same two classes using the MIS-only method was approximately $91 \%$. This was under broadly similar experimental conditions with the following exceptions: (1) Kutila et al. used real scrap metal, as opposed to manufactured test pieces in the present work, (2) a larger mass of waste was 


\begin{tabular}{|c|c|c|c|c|c|c|c|c|c|c|c|c|}
\hline Classifier & Features & $i$ & $\mathrm{Tp}_{i}$ & $\mathrm{Fn}_{i}$ & $\operatorname{Tn}_{i}$ & $\mathrm{Fp}_{i}$ & Acc. $_{i}$ & Prec $_{. i}$ & Rec. $_{i}$ & Acc. $\mu$ & Prec. $\mu$ & Rec. $\mu$ \\
\hline Logistic function & $Z_{64 k H z}^{\prime}, Z_{4 k H z}^{\prime \prime}$ & $\begin{array}{l}\mathrm{Cu} \\
\mathrm{Al} \\
\mathrm{Br}\end{array}$ & $\begin{array}{l}36 \\
37 \\
35\end{array}$ & $\begin{array}{l}0 \\
7 \\
2\end{array}$ & $\begin{array}{l}75 \\
71 \\
79\end{array}$ & $\begin{array}{l}6 \\
2 \\
1\end{array}$ & $\begin{array}{l}0.9487 \\
0.9231 \\
0.9744\end{array}$ & $\begin{array}{l}0.8571 \\
0.9487 \\
0.9722\end{array}$ & $\begin{array}{l}1.0000 \\
0.8409 \\
0.9459\end{array}$ & 0.9487 & 0.9231 & 0.9231 \\
\hline Sample Matching & $\begin{array}{c}Z^{\prime}, Z^{\prime \prime} \text { at all freqs., } \\
f_{1}, f_{2}, f_{3}, f_{4}, \\
K=5 \text { (i.e. size of } U_{K}^{i} \text { ) }\end{array}$ & $\begin{array}{l}\mathrm{Cu} \\
\mathrm{Al} \\
\mathrm{Br}\end{array}$ & $\begin{array}{l}26 \\
31 \\
31\end{array}$ & $\begin{array}{c}10 \\
13 \\
6\end{array}$ & $\begin{array}{l}72 \\
57 \\
76\end{array}$ & $\begin{array}{c}9 \\
16 \\
4\end{array}$ & $\begin{array}{l}0.8376 \\
0.7521 \\
0.9145\end{array}$ & $\begin{array}{l}0.7429 \\
0.6596 \\
0.8857\end{array}$ & $\begin{array}{l}0.7222 \\
0.7045 \\
0.8378\end{array}$ & 0.8348 & 0.7521 & 0.7521 \\
\hline
\end{tabular}

TABLE I: Classification statistics

tested (over $4000 \mathrm{~kg}$ ), and (3) a faster conveyor speed of 1.5 $\mathrm{m} / \mathrm{s}$ compared to $1 \mathrm{~m} / \mathrm{s}$ in this work. The use of manufactured rather than real scrap test pieces may artificially inflate the performance statistics of the MIS classifiers, however, the latter two differences are unlikely to make much difference. Overall, Kutila et al. concluded that induction was not much use in sorting these metal types, however we find otherwise when using a multi-frequency approach.

Hyper-spectral techniques have shown high recall around 90-97\% [13]. Picon et al [12] reported an average recall of $97.63 \%$ for separating brass, copper and aluminium. Candiani et al. [11] reported $92-100 \%$ for the same metals. In comparison, we report average recall around $92 \%$ for the MIS-only classifiers. The main differences in experimental conditions were: (1) The hyper-spectral studies used a small set of handselected real scrap. (2) In Candiani et al., the samples were significantly smaller - less than $2 \mathrm{~mm}$ in size, and (3) the hyper-spectral studies used much slower belt-speeds - 0.33 $\mathrm{m} / \mathrm{s}$ (Picon et al.) and $0.067 \mathrm{~m} / \mathrm{s}$ (Candiani et al.) versus 1 $\mathrm{m} / \mathrm{s}$ in the present study. Commercial belts typically operate in excess of $2.5 \mathrm{~m} / \mathrm{s}$, hence higher belt-speeds are needed for realistic comparison.

\section{CONCLUSION}

We have demonstrated using magnetic induction spectroscopy and simple geometric features to classify the main non-ferrous metals found in end-of-life waste (copper, aluminium and brass). Our method has the advantages of being fast and scalable with relatively modest measurement hardware. The performance of the MIS-only classifiers were particularly promising, with around $92 \%$ precision and recall (equivalent to purity and recovery-rate). This is within industry prescribed thresholds and broadly competitive with reported statistics for comparable systems.

A surprising result was the relatively low performance of the combined vision and MIS classifier. We speculate that simple geometric features (area, height, max./min. feret diameter) fail to capture the information required to make meaningful predictions about the scattered magnetic field - illustrated by poor banding between metals shown in figure 8 . The component $Z_{64 k \mathrm{~Hz}}^{\prime}$ was found to be a superior geometric feature in this respect. We do not suggest that vision data has no value, rather that its interpretation demands a more thorough treatment beyond the scope of the present work.
Induction sensors are distinctly applicable to metal recycling: They are low-cost, reliable, practical, and robust. They are unaffected by variable lighting or dirty samples, and resistant to harsh environments. However, their performance is dependent on a degree of homogeneity in the geometry of the metal fragments; something difficult to achieve in practice.

Our main conclusion is that an effective non-ferrous metal sorting system can be constructed using multi-frequency induction sensors and a simple, straightforward classification algorithm. The most novel aspect is the use of the magnetic induction spectra and the selection of frequency components as features, based on the physics of how induced eddy-currents circulate in metal objects. Two frequency components are chosen as features: (1) A high frequency component, where magnetic field penetration is negligible, to model the geometry of the metal fragments, and (2) a low frequency component, where skin-depth is significant, which is a function of both geometry and conductivity of the fragments. We have shown that, provided these key criteria are satisfied, good performance rates can be achieved, and a simple but effective classifier can be created distinctly well-suited to industry needs.

\section{ACKNOWLEDGMENT}

This research was conducted as part of the EU FP7 funded project Shreddersort, under grant agreement no. 603676. We would especially like to acknowledge Alfred Rinhofer and Malte Jaschik of Joanneum Research for their on-going support with the vision system, and Lenz Instruments, in particular Mark Williams, for integration of the industrial prototype.

\section{APPENDIX}

The headings for table I, with $C:=\{C u, A l, B r\}$ :

- $T p_{i}, F n_{i}, T n_{i}, F p_{i}$ : Number of true-positives, falsenegatives, true-negatives, and false-positives for class $i$.

- $A c c_{i}$ : Accuracy for class $i$, i.e. proportion of pieces in class $i$, correctly assigned class $i(T p)$ or not class $i(T n)$,

$$
A c c_{i}:=\left(T p_{i}+T n_{i}\right) /\left(T p_{i}+F n_{i}+F p_{i}+T n_{i}\right)
$$

- $P r e c_{i}$ : Precision for class $i$, i.e. the proportion of pieces assigned class $i$ that actually belong to that class,

$$
\operatorname{Prec}_{i}:=T p_{i} /\left(T p_{i}+F p_{i}\right)
$$

- $R e c_{i}$ : Recall or recovery-rate for class $i$, i.e. the proportion of pieces in class $i$ correctly assigned to that class,

$$
R e c_{i}:=T p_{i} /\left(T p_{i}+F n_{i}\right)
$$


- $A c c_{\mu}$ : Average accuracy, $\sum_{i \in C} A c c_{i} / 3$

- $\operatorname{Prec}_{\mu}$ : Av. precision, $\sum_{i \in C} T p_{i} / \sum_{i \in C}\left(T p_{i}+F p_{i}\right)$

- $R e c_{\mu}$ : Av. recall, $\sum_{i \in C} T p_{i} / \sum_{i \in C}\left(T p_{i}+F n_{i}\right)$

\section{REFERENCES}

[1] S.-i. Sakai et al., "An international comparative study of end-of-life vehicle (elv) recycling systems," Journal of Material Cycles and Waste Management, vol. 16, no. 1, pp. 1-20, Feb 2014.

[2] "Directive 2000/53/EC of the European Parliament and of the Council of 18 September 2000 on End-of-Life vehicles," Official Journal of the European Communities, vol. 43, 2000.

[3] Eurostat, 2014 "End-of-life vehicles - reuse, recycling and recovery of ELVS", Last update 11/09/2017.

[4] O. Forton, M. Harder, and N. Moles, "Value from shredder waste: Ongoing limitations in the uk," Resources, Conservation and Recycling, vol. 46, no. 1, pp. $104-113,2006$.

[5] A. Santini, L. Morselli, F. Passarini, I. Vassura, S. D. Carlo, and F. Bonino, "End-of-life vehicles management: Italian material and energy recovery efficiency," Waste Management, vol. 31, no. 3, pp. 489 $-494,2011$.

[6] Inst. of Scrap Recycling Industries, "ISRI Scrap Specifications, Circular 1994: Guidelines for Nonferrous Scrap, Ferrous Scrap, Glass Cullet, Paper Stock, Plastic Scrap", 1994.

[7] F. Margarido, R. N. Santos, F. Durão, C. Guimarães, C. Nogueira, P. C. Oliveira, F. Pedrosa, and A. M. Gonçalves, "Separation of nonferrous fractions of shredded end-of-life vehicles for valorising its alloys," in MMME'14-International Conference on Mining, Material and Metallurgical Engineering, 2014.

[8] W. L. Dalmijn and T. P. R. De Jong, "The development of vehicle recycling in europe: Sorting, shredding, and separation," JOM, vol. 59, no. 11, pp. 52-56, Nov 2007.

[9] G. Gaustad, E. Olivetti, and R. Kirchain, "Improving aluminium recycling: A survey of sorting and impurity removal technologies," Resources, Conservation and Recycling, vol. 58, pp. 79-87, 2012.

[10] M. Kutila, J. Viitanen, and A. Vattulainen, "Scrap metal sorting with colour vision and inductive sensor array," in International Conference on Computational Intelligence for Modelling, Control and Automation and International Conference on Intelligent Agents, Web Technologies and Internet Commerce (CIMCA-IAWTIC'06), vol. 2, Nov 2005, pp. $725-729$.

[11] G. Candiani, N. Picone, L. Pompilio, M. Pepe, and M. Colledani, "Characterization of fine metal particles derived from shredded weee using a hyperspectral image system: Preliminary results," Sensors, vol. 17, no. 5, 2017.

[12] A. Picon, O. Ghita, P. F. Whelan, and P. M. Iriondo, "Fuzzy spectral and spatial feature integration for classification of nonferrous materials in hyperspectral data," IEEE Transactions on Industrial Informatics, vol. 5, no. 4, pp. 483-494, Nov 2009.

[13] S. P. Gundupalli, S. Hait, and A. Thakur, "A review on automated sorting of source-separated municipal solid waste for recycling," Waste Management, vol. 60, pp. 56 - 74, 2017.

[14] J. R. Wait, "A conducting permeable sphere in the presence of a coil carrying an oscillating current," Canadian Journal of Physics, vol. 31, no. 4, pp. 670-678, 1953.

[15] K. Sun, K. O’Neill, F. Shubitidze, S. A. Haider, and K. D. Paulsen, "Simulation of electromagnetic induction scattering from targets with negligible to moderate penetration by primary fields," IEEE Trans. on Geoscience and Remote Sensing, vol. 40, no. 4, pp. 910-927, Apr 2002.

[16] J. R. Wait, "Some solutions for electromagnetic problems involving spheroidal, spherical, and cylindrical bodies," Res. NBS (Math. and Mathematical Physics) B, vol. 64, pp. 15-32, 1959.

[17] M. D. O'Toole, J. L. Davidson, L. A. Marsh, W. Yin, and A. J. Peyton, "Evaluation of the thin-skin approximation boundary element method for electromagnetic induction scattering problems," in 2016 IEEE Sensors Applications Symposium (SAS), April 2016, pp. 1-6.

[18] O. A. Abdel-Rehim, J. L. Davidson, L. A. Marsh, M. D. OToole, and A. J. Peyton, "Magnetic polarizability tensor spectroscopy for low metal anti-personnel mine surrogates," IEEE Sensors Journal, vol. 16, no. 10, pp. 3775-3783, May 2016.

[19] Uni. of Manchester, "Method and apparatus for determining conductivity," UK Patent Application Number GB1 706 951.9.

[20] M. D. O'Toole, L. A. Marsh, J. L. Davidson, Y. M. Tan, D. W. Armitage, and A. J. Peyton, "Non-contact multi-frequency magnetic induction spectroscopy system for industrial-scale bio-impedance measurement," Measurement Science and Technology, vol. 26, no. 3, p. 035102, 2015.
[21] A. K. Jain and R. C. Dubes, Algorithms for Clustering Data. Upper Saddle River, NJ, USA: Prentice-Hall, Inc., 1988.

[22] M. D. O'Toole, N. Karimian, and A. J. Peyton, "Fast classification of non-magnetic metal targets using eddy-current based impedance spectroscopy - to be published," in 2017 IEEE SENSORS, Oct 2017.

[23] M. Sokolova and G. Lapalme, "A systematic analysis of performance measures for classification tasks," Information Processing \& Management, vol. 45, no. 4, pp. 427 - 437, 2009.

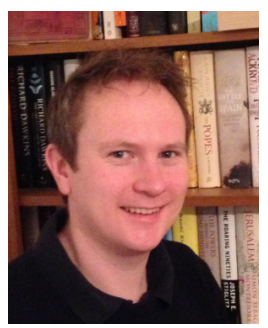

Michael D O'Toole received the M.Eng. (Hons.) degree in integrated engineering from the University of Reading in 2006, and the Ph.D. degree from the Wolfson School of Mechanical and Manufacturing Engineering, Loughborough University, in 2011. He was a Research Associate at The University of Manchester from 2011 to 2016, first working in the School of Computer Science on simulation and analysis tools for nonsmooth mechanical systems then in School of Electrical and Electronic Engineering from 2012 working on magnetic induction systems for non-destructive inspection and characterisation.

In 2016, he was awarded a Leverhulme Trust Early Career Research Fellowship. He is author and co-author of over 20 scientific publications and has recently filed his first patent. His research interests include signal processing, and sensor and instrumentation design, with a particular emphasis toward bioimpedance spectroscopy and magnetic induction systems for nondestructive testing.

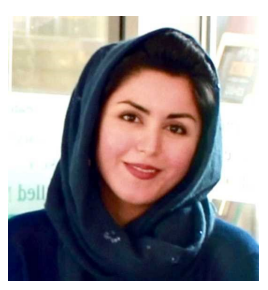

Noushin Karimian received her Ph.D. degree in Electrical and Electronic Engineering from the University of Manchester in 2014. Since then she has been appointed as a Research Associate at this university on various international projects. Her main research interests are in Electromagnetic sensors for NDT applications and digital signal processing. She has been actively involved both as technical and organisational committee member of international conferences, and most recently the European Microwave Conference. She is co-author of over 40 international conference and journal papers and has a registered patent.

Dr Karimian is a member of IET, EuMA, BINDT and IOP. She is currently acting as the Treasurer for the IEEE UK \& Ireland Section, and a committee member of the University of Manchester IEEE Student Branch. She is also a committee member of the IEEE UK \& Ireland Women in Engineering (WiE).

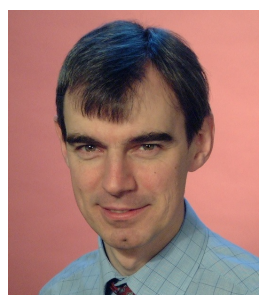

Anthony J Peyton received the B.Sc. degree in electrical engineering and electronics and the Ph.D. degree in medical instrumentation from UMIST in 1983 and 1986, respectively. He was appointed Principal Engineer at Kratos Analytical Ltd. in 1989, developing precision electronic instrumentation systems for magnetic sector and quadrupole mass spectrometers, from which an interest in electromagnetic instrumentation developed. He returned to UMIST as a Lecturer and worked with the Process Tomography Group. He moved to Lancaster University in 1996 as Senior Lecturer. He was promoted to Reader in electronic instrumentation in 2001 and Professor in May 2004. Since December 2004, he has been a Professor of Electromagnetic Tomography Engineering with the University of Manchester. His main research interests currently are in the area of instrumentation, applied sensor systems, and electromagnetics. He has been a Principal Investigator of numerous national and industry funded projects and a partner of ten previous EU projects, one as a Coordinator. He has been a coauthor of over 110 international journal papers, two books, several hundred conference papers, and 12 patents in areas related to electromagnetics and tomography. 